\title{
Adsorption of Long Straight Rigid Rods on Two- Dimensional Lattices: Study of Orientational Surface Phase Transitions from Entropic Considerations ${ }^{\dagger}$
}

\author{
Eugenio Vogel 1,2, Julian J. Riccardo ${ }^{3}$, José L. Riccardo ${ }^{3}$, Pedro Marcelo Pasinetti ${ }^{3}$ and \\ Antonio José Ramirez-Pastor ${ }^{3}$ \\ 1 Department of Physics, Universidad de La Frontera, Temuco, Chile \\ 2 Centro para el Desarrollo de la Nanociencia y la Nanotecnología, CEDENNA, Santiago, Chile \\ 3 Departamento de Física, Instituto de Física Aplicada, Universidad Nacional de San Luis-CONICET, \\ Ejército de los Andes 950, D5700BWS, San Luis, Argentina \\ † Presented at the Entropy 2021: The Scientific Tool of the 21st Century, 5-7 May 2021; Available online: \\ https://sciforum.net/conference/Entropy2021/.
}

Published: 5 May 2021

The orientational phase transitions occurring in a system of long straight rigid rods of length $k(k-$ mers) on square lattices are studied by combining Monte Carlo simulations and theoretical analysis. The phenomenology of this model was examined in Refs. [1-5]. A nematic (N) phase, characterized by a big domain of parallel $k$-mers, is separated from a disordered-isotropic (D) state, by a continuous transition occurring at intermediate density. A second phase transition, from an $\mathrm{N}$ order to an orderedisotropic $(\mathrm{O})$ state, occurs near saturation density values. In the present work, the process is analyzed by following the number of accessible adsorption states along the vertical[horizontal] direction as a function of the surface coverage $\mathrm{W}_{\mathrm{v}}(\mathrm{q})\left[\mathrm{W}_{\mathrm{h}}(\mathrm{q})\right]$, which allows us to define a vertical[horizontal] configurational entropy. These quantities show strong variations with coverage (eventually leading to ergodicity breakdown), allowing to identify the different phases ( $N, \mathrm{D}$ and $\mathrm{O}$ ) characterizing the critical behavior of the system. Comparisons between Monte Carlo simulations and analytical calculations were performed in order to evaluate the reaches and limitations of the theoretical model.

\section{References}

1. Ghosh, A.; Dhar, D. On the orientational ordering of long rods on a lattice. EPL (Europhys. Lett.) 2007, 78, 20003.

2. Matoz-Fernandez, D.A.; Linares, D.H.; Ramirez-Pastor, A.J. Determination of the critical exponents for the isotropic-nematic phase transition in a system of long rods on two-dimensional lattices: Universality of the transition. EPL (Europhys. Lett.) 2008, 82, 50007.

3. Kundu, J.; Rajesh, R.; Dhar, D.; Stilck, J.F. Nematic-disordered phase transition in systems of long rigid rods on two-dimensional lattices. Phys. Rev. E 2013, 87, 032103.

4. Vogel, E.E.; Saravia, G.; Ramirez-Pastor, A.J. Phase transitions in a system of long rods on two-dimensional lattices by means of information theory. Phys. Rev. E 2017, 96, 062133.

5. Vogel, E.E.; Saravia, G.; Ramirez-Pastor, A.J.; Pasinetti, M. Alternative characterization of the nematic transition in deposition of rods on two-dimensional lattices. Phys. Rev. E 2020, 101, 022104.

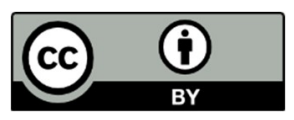

(C) 2021 by the authors. Licensee MDPI, Basel, Switzerland. This article is an open access article distributed under the terms and conditions of the Creative Commons Attribution (CC BY) license (http://creativecommons.org/licenses/by/4.0/). 